\title{
On subclasses of bi-convex functions defined by Tremblay fractional derivative operator
}

Sevtap Sümer Eker and Bilal Şeker

\begin{abstract}
We introduce and investigate new subclasses of analytic and biunivalent functions defined by modified Tremblay operator in the open unit disk. Also we obtain upper bounds for the coefficients of functions belonging to these classes.
\end{abstract}

Mathematics Subject Classification (2010): 30C45, 30C50, 30C80.

Keywords: Bi-convex functions, Tremblay fractional derivative, coefficient bounds and coefficient estimates.

\section{Introduction}

Let $\mathcal{A}$ denote the class of functions $f(z)$ which are analytic in the open unit disk $\mathbb{U}=\{z: z \in \mathbb{C}$ and $|z|<1\}$ and normalized by the conditions $f(0)=f^{\prime}(0)-1=0$ and having the form:

$$
f(z)=z+\sum_{n=2}^{\infty} a_{n} z^{n} .
$$

Also let $\mathcal{S}$ denote the subclass of functions in $\mathcal{A}$ which are univalent in $\mathbb{U}$ (for details, see [7]).

The Koebe One Quarter Theorem (e.g., see [7]) ensures that the image of $\mathbb{U}$ under every univalent function $f(z) \in \mathcal{A}$ contains the disk of radius $1 / 4$. Thus every univalent function $f$ has an inverse $f^{-1}$ satisfying

$$
f^{-1}(f(z))=z \quad(z \in \mathbb{U})
$$

and

$$
f\left(f^{-1}(w)\right)=w \quad\left(|w|<r_{0}(f), r_{0}(f) \geq \frac{1}{4}\right)
$$


In fact, the inverse function $f^{-1}$ is given by

$$
\begin{aligned}
g(w) & =f^{-1}(w)=w-a_{2} w^{2}+\left(2 a_{2}^{2}-a_{3}\right) w^{3}-\left(5 a_{2}^{3}-5 a_{2} a_{3}+a_{4}\right) w^{4}+\cdots \\
& =w+\sum_{n=2}^{\infty} b_{n} w^{n} .
\end{aligned}
$$

A function $f \in \mathcal{A}$ is said to be bi-univalent in $\mathbb{U}$ if both $f(z)$ and $f^{-1}(z)$ are univalent in $\mathbb{U}$. We denote by $\Sigma$ the class of all bi-univalent functions in $\mathbb{U}$ given by the TaylorMaclaurin series expansion (1.1).

For a brief history and examples of functions in the class $\Sigma$, see [19] (see also $[5],[6],[11],[25])$.

Coefficient bounds for various subclasses of bi-univalent functions were obtained by several authors including Ali et al. [2], Caglar et al. [3], Deniz [4], Kumar et al. [10], Magesh and Yamini [12], Srivastava et al. [17], [18], [22], Sümer Eker [1], [23], [24]. In fact, judging by the remarkable flood of papers on the subject, the pioneering work of Srivastava et al. [19] appears to have revived the study of analytic and bi-univalent functions in recent years.

The following definition of fractional derivative will be required in our investigation (see, for details, [13], [14], [20], [21]).

Definition 1.1. The fractional integral of order $\delta$ is defined, for a function $f$, by

$$
D_{z}^{-\delta} f(z)=\frac{1}{\Gamma(\delta)} \int_{0}^{z} \frac{f(\xi)}{(z-\xi)^{1-\delta}} d \xi ;(\delta>0),
$$

where $f$ is an analytic function in a simply-connected region of complex $z$-plane containing the origin, and the multiplicity of $(z-\xi)^{\delta-1}$ is removed by requiring, $\log (z-\xi)$ to be real when $z-\xi>0$.

Definition 1.2. The fractional derivative of order $\delta$ is defined, for a function $f$, by

$$
D_{z}^{\delta} f(z)=\frac{1}{\Gamma(1-\delta)} \frac{d}{d z} \int_{0}^{z} \frac{f(\xi)}{(z-\xi)^{\delta}} d \xi(0 \leq \delta<1),
$$

where $f$ is constrained, and the multiplicity of $(z-\xi)^{-\delta}$ is removed, as in Definition 1.1 .

Definition 1.3. Under the hypotheses of Definition 2, the fractional derivative of order $(n+\delta)$ is defined by

$$
D_{z}^{n+\delta} f(z)=\frac{d^{n}}{d z^{n}} D_{z}^{\delta} f(z) \quad\left(0 \leq \delta<1, n \in \mathbb{N}_{0}=\mathbb{N} \cup\{0\}\right)
$$

By virtue of Definitions 1.1, 1.2 and 1.3, we have

$$
D_{z}^{-\delta} z^{n}=\frac{\Gamma(n+1)}{\Gamma(n+\delta+1)} z^{n+\delta} \quad(n \in \mathbb{N}, \delta>0)
$$

and

$$
D_{z}^{\delta} z^{n}=\frac{\Gamma(n+1)}{\Gamma(n-\delta+1)} z^{n-\delta} \quad(n \in \mathbb{N}, 0 \leq \delta<1)
$$


Tremblay [26] studied a fractional calculus operator defined in terms of the RiemannLiouville fractional differential operator. Ibrahim and Jahangiri [9] extended and studied this operator in the complex plane.

Definition 1.4. The Tremblay fractional derivative operator $\mathrm{T}_{z}^{\mu, \gamma}$ of a function $f \in \mathcal{A}$ is defined, for all $z \in \mathbb{U}$, by

$$
\begin{gathered}
\mathrm{T}_{z}^{\mu, \gamma} f(z)=\frac{\Gamma(\gamma)}{\Gamma(\mu)} z^{1-\gamma} D_{z}^{\mu-\gamma} z^{\mu-1} f(z) \\
(0<\mu \leq 1 ; 0<\gamma \leq 1 ; \mu \geq \gamma ; 0 \leq \mu-\gamma<1) .
\end{gathered}
$$

It is clear that, for $\mu=\gamma=1$, we have

$$
\mathrm{T}_{z}^{1,1} f(z)=f(z)
$$

Example 1.5. Let $f(z)=z^{n}$. The Tremblay Fractional Derivative of $f(z)$ is:

$$
\mathrm{T}_{z}^{\mu, \gamma} f(z)=\frac{\Gamma(\gamma)}{\Gamma(\mu)} \frac{\Gamma(n+\mu)}{\Gamma(n+\gamma)} z^{n}
$$

and for $\mu=\gamma=1$, we have $\mathrm{T}_{z}^{1,1}\left(z^{n}\right)=z^{n}$.

Recently in [8], Esa et al. defined modified of Tremblay operator of analytic functions in complex domain as follows:

Definition 1.6. Let $f(z) \in \mathcal{A}$. The modified Tremblay operator denoted by $\mathfrak{T}^{\mu, \gamma}: \mathcal{A} \rightarrow$ $\mathcal{A}$ and defined such as:

$$
\begin{aligned}
\mathfrak{T}^{\mu, \gamma} f(z) & =\frac{\gamma}{\mu} \mathrm{T}_{z}^{\mu, \gamma} f(z) \\
& =\frac{\Gamma(\gamma+1)}{\Gamma(\mu+1)} z^{1-\gamma} D_{z}^{\mu-\gamma} z^{\mu-1} f(z) \\
& =z+\sum_{n=2}^{\infty} \frac{\Gamma(\gamma+1) \Gamma(n+\mu)}{\Gamma(\mu+1) \Gamma(n+\gamma)} a_{n} z^{n}
\end{aligned}
$$

The object of the present paper is to introduce a new subclass of the function class $\Sigma$ by using the modified Tremblay operator and find estimate on the coefficients $\left|a_{2}\right|$ and $\left|a_{3}\right|$ for functions in this class.

We begin by introducing the function class $\mathcal{C}_{\Sigma}^{\mu, \gamma}(\alpha)$ by means of the following definition.

\section{Main results}

Definition 2.1. A function $f(z)$ given by (1.1) is said to be in the class $\mathcal{C}_{\Sigma}^{\mu, \gamma}(\alpha)$ $(0<\mu \leq 1 ; 0<\gamma \leq 1 ; \mu \geq \gamma ; 0 \leq \mu-\gamma<1)$ if the following conditions are satisfied:

$$
f \in \Sigma \text { and }\left|\arg \left(1+\frac{z(\mathfrak{T} f)^{\prime \prime}(z)}{\mathfrak{T} f^{\prime}(z)}\right)\right|<\frac{\alpha \pi}{2} \quad(0<\alpha \leq 1, z \in \mathbb{U})
$$

and

$$
\left|\arg \left(1+\frac{w(\mathfrak{T} g)^{\prime \prime}(w)}{\mathfrak{T} g^{\prime}(w)}\right)\right|<\frac{\alpha \pi}{2} \quad(0<\alpha \leq 1, w \in \mathbb{U})
$$


where the function $g(w)$ is given by (1.2).

We first state and prove the estimates on the coefficients $\left|a_{2}\right|$ and $\left|a_{3}\right|$ for functions in the class $\mathcal{C}_{\Sigma}^{\mu, \gamma}(\alpha)$.

Theorem 2.2. If $f(z)$ given by (1.1) be in the class $\mathcal{C}_{\Sigma}^{\mu, \gamma}(\alpha)$, then

$$
\left|a_{2}\right| \leq \alpha(\gamma+1) \sqrt{\frac{(\gamma+2)}{(\mu+1)[3 \alpha(\mu+2)(\gamma+1)+(1-3 \alpha)(\mu+1)(\gamma+2)]}}
$$

and

$$
\left|a_{3}\right| \leq \frac{\alpha(\gamma+2)(\gamma+1)^{2}}{(\mu+1)(\mu \gamma-\mu+4 \gamma+2)}
$$

Proof. For $f$ given by (1.1), we can write from (2.1) and (2.2)

$$
\begin{gathered}
1+\frac{z(\mathfrak{T} f)^{\prime \prime}(z)}{\mathfrak{T} f^{\prime}(z)}=[p(z)]^{\alpha} \\
1+\frac{w(\mathfrak{T} g)^{\prime \prime}(w)}{\mathfrak{T} g^{\prime}(w)}=[q(w)]^{\alpha}
\end{gathered}
$$

where $p(z)$ and $q(w)$ are in familiar Caratheódory Class $\mathcal{P}$ (see for details [7]) and have the following series representations:

$$
p(z)=1+p_{1} z+p_{2} z^{2}+p_{3} z^{3}+\cdots
$$

and

$$
q(w)=1+q_{1} w+q_{2} w^{2}+q_{3} w^{3}+\cdots
$$

Now, equating the coefficients (2.5) and (2.6), we find that

$$
\begin{gathered}
2 \frac{\mu+1}{\gamma+1} a_{2}=\alpha p_{1}, \\
\frac{6(\mu+2)(\mu+1)}{(\gamma+2)(\gamma+1)} a_{3}-4\left(\frac{\mu+1}{\gamma+1}\right)^{2} a_{2}^{2}=\alpha p_{2}+\frac{\alpha(\alpha-1)}{2} p_{1}^{2} \\
-2 \frac{\mu+1}{\gamma+1} a_{2}=\alpha q_{1}
\end{gathered}
$$

and

$$
\frac{6(\mu+2)(\mu+1)}{(\gamma+2)(\gamma+1)}\left(2 a_{2}^{2}-a_{3}\right)-4\left(\frac{\mu+1}{\gamma+1}\right)^{2} a_{2}^{2}=\alpha q_{2}+\frac{\alpha(\alpha-1)}{2} q_{1}^{2} .
$$

From (2.9) and (2.11), we get

and

$$
p_{1}=-q_{1}
$$

$$
8\left(\frac{\mu+1}{\gamma+1}\right)^{2} a_{2}^{2}=\alpha^{2}\left(p_{1}^{2}+q_{1}^{2}\right)
$$

Also from (2.10), (2.12) and 2.14, we get

$$
a_{2}^{2}=\frac{\alpha^{2}\left(p_{2}+q_{2}\right)(\gamma+2)(\gamma+1)^{2}}{4(\mu+1)[3 \alpha(\mu+2)(\gamma+1)+(1-3 \alpha)(\mu+1)(\gamma+2)]} .
$$


According to the Caratheódory Lemma (see [7]), $\left|p_{n}\right| \leq 2$ and $\left|q_{n}\right| \leq 2$ for $n \in \mathbb{N}$. Now taking the absolute value of (2.15) and applying the Carathéodory Lemma for coefficients $p_{2}$ and $q_{2}$ we obtain

$$
\left|a_{2}\right| \leq \sqrt{\frac{\alpha^{2}(\gamma+2)(\gamma+1)^{2}}{(\mu+1)[3 \alpha(\mu+2)(\gamma+1)+(1-3 \alpha)(\mu+1)(\gamma+2)]}} .
$$

This gives desired bound for $\left|a_{2}\right|$ as asserted in (2.3).

Now, in order to find the bound on $\left|a_{3}\right|$, from (2.12) and (2.10) and (2.13), we can write

$$
\begin{gathered}
\left\{\frac{72(\mu+2)^{2}(\mu+1)^{2}}{(\gamma+2)^{2}(\gamma+1)^{2}}-\frac{48(\mu+2)(\mu+1)^{3}}{(\gamma+2)(\gamma+1)^{3}}\right\} a_{3} \\
=\alpha\left\{\left(\frac{12(\mu+2)(\mu+1)}{(\gamma+2)(\gamma+1)}-\frac{4(\mu+1)^{2}}{(\gamma+1)^{2}}\right) p_{2}+\frac{4(\mu+1)^{2}}{(\gamma+1)^{2}} q_{2}\right\} \\
+\frac{6 \alpha(\alpha-1)(\mu+2)(\mu+1)}{(\gamma+2)(\gamma+1)} p_{1}^{2} .
\end{gathered}
$$

If $\alpha=1$ then

$$
\left|a_{3}\right| \leq \frac{(\gamma+2)(\gamma+1)^{2}}{(\mu+1)(\mu \gamma-\mu+4 \gamma+2)}
$$

Now, we consider the case $0<\alpha<1$. From (2.16), we can write

$$
\begin{gathered}
\left\{\frac{72(\mu+2)^{2}(\mu+1)^{2}}{(\gamma+2)^{2}(\gamma+1)^{2}}-\frac{48(\mu+2)(\mu+1)^{3}}{(\gamma+2)(\gamma+1)^{3}}\right\} \operatorname{Re}\left(a_{3}\right) \\
=\alpha \operatorname{Re}\left\{\left(\frac{12(\mu+2)(\mu+1)}{(\gamma+2)(\gamma+1)}-\frac{4(\mu+1)^{2}}{(\gamma+1)^{2}}\right) p_{2}+\frac{4(\mu+1)^{2}}{(\gamma+1)^{2}} q_{2}\right\} \\
+\operatorname{Re} \frac{6 \alpha(\alpha-1)(\mu+2)(\mu+1)}{(\gamma+2)(\gamma+1)} p_{1}^{2} .
\end{gathered}
$$

From Herglotz's Representation formula [15] for the functions $p(z)$ and $q(w)$, we have

$$
p(z)=\int_{0}^{2 \pi} \frac{1+z e^{-i t}}{1-z e^{-i t}} d \mu_{1}(t)
$$

and

$$
q(w)=\int_{0}^{2 \pi} \frac{1+w e^{-i t}}{1-w e^{-i t}} d \mu_{2}(t)
$$

where $\mu_{i}(t)$ are increasing on $[0,2 \pi]$ and $\mu_{i}(2 \pi)-\mu_{i}(0)=1, i=1,2$.

We also have

$$
\begin{array}{ll}
p_{n}=2 \int_{0}^{2 \pi} e^{-i n t} d \mu_{1}(t), & n=1,2, \ldots \\
q_{n}=2 \int_{0}^{2 \pi} e^{-i n t} d \mu_{2}(t), & n=1,2, \ldots
\end{array}
$$

Now (2.17) can be written as follows:

$$
\left\{\frac{72(\mu+2)^{2}(\mu+1)^{2}}{(\gamma+2)^{2}(\gamma+1)^{2}}-\frac{48(\mu+2)(\mu+1)^{3}}{(\gamma+2)(\gamma+1)^{3}}\right\} \operatorname{Re}\left(a_{3}\right)
$$




$$
\begin{gathered}
=\alpha\left\{\left(\frac{12(\mu+2)(\mu+1)}{(\gamma+2)(\gamma+1)}-\frac{4(\mu+1)^{2}}{(\gamma+1)^{2}}\right) 2 \int_{0}^{2 \pi} \cos 2 t d \mu_{1}(t)+\frac{8(\mu+1)^{2}}{(\gamma+1)^{2}} \int_{0}^{2 \pi} \cos 2 t d \mu_{2}(t)\right\} \\
-\frac{24 \alpha(1-\alpha)(\mu+2)(\mu+1)}{(\gamma+2)(\gamma+1)}\left[\left(\int_{0}^{2 \pi} \cos t d \mu_{1} t\right)^{2}-\left(\int_{0}^{2 \pi} \sin t d \mu_{1}(t)\right)^{2}\right] \\
\leq 2 \alpha\left\{\left(\frac{12(\mu+2)(\mu+1)}{(\gamma+2)(\gamma+1)}-\frac{4(\mu+1)^{2}}{(\gamma+1)^{2}}\right) \int_{0}^{2 \pi} \cos 2 t d \mu_{1}(t)+\frac{4(\mu+1)^{2}}{(\gamma+1)^{2}} \int_{0}^{2 \pi} \cos 2 t d \mu_{2}(t)\right\} \\
+\frac{24 \alpha(1-\alpha)(\mu+2)(\mu+1)}{(\gamma+2)(\gamma+1)}\left(\int_{0}^{2 \pi} \sin t d \mu_{1}(t)\right)^{2} \\
=2 \alpha\left(\frac{12(\mu+2)(\mu+1)}{(\gamma+2)(\gamma+1)}-\frac{4(\mu+1)^{2}}{(\gamma+1)^{2}}\right) \int_{0}^{2 \pi}\left(1-2 \sin ^{2} t\right) d \mu_{1}(t) \\
+\frac{8 \alpha(\mu+1)^{2}}{(\gamma+1)^{2}} \int_{0}^{2 \pi}\left(1-2 \sin ^{2} t\right) d \mu_{2}(t)+\frac{24 \alpha(1-\alpha)(\mu+2)(\mu+1)}{(\gamma+2)(\gamma+1)}\left(\int_{0}^{2 \pi} \sin t d \mu_{1}(t)\right)^{2} .
\end{gathered}
$$

By Jensen's inequality ([16]), we have

$$
\left(\int_{0}^{2 \pi}|\sin t| d \mu(t)\right)^{2} \leq \int_{0}^{2 \pi} \sin ^{2} t d \mu(t) .
$$

Hence

$$
\begin{gathered}
\left\{\frac{72(\mu+2)^{2}(\mu+1)^{2}}{(\gamma+2)^{2}(\gamma+1)^{2}}-\frac{48(\mu+2)(\mu+1)^{3}}{(\gamma+2)(\gamma+1)^{3}}\right\} \operatorname{Re}\left(a_{3}\right) \\
\leq 2 \alpha\left(\frac{12(\mu+2)(\mu+1)}{(\gamma+2)(\gamma+1)}-\frac{4(\mu+1)^{2}}{(\gamma+1)^{2}}\right) \\
-4 \alpha\left(\frac{12(\mu+2)(\mu+1)}{(\gamma+2)(\gamma+1)}-\frac{4(\mu+1)^{2}}{(\gamma+1)^{2}}\right) \int_{0}^{2 \pi} \sin ^{2} t d \mu_{1}(t) \\
+\frac{8 \alpha(\mu+1)^{2}}{(\gamma+1)^{2}}-\frac{16 \alpha(\mu+1)^{2}}{(\gamma+1)^{2}} \int_{0}^{2 \pi} \sin ^{2} t d \mu_{2}(t) \\
+\frac{24 \alpha(1-\alpha)(\mu+2)(\mu+1)}{(\gamma+2)(\gamma+1)} \int_{0}^{2 \pi} \sin ^{2} t d \mu_{1}(t)
\end{gathered}
$$

and thus

$$
\operatorname{Re}\left(a_{3}\right) \leq \frac{\alpha(\gamma+2)(\gamma+1)^{2}}{(\mu+1)(\mu \gamma-\mu+4 \gamma+2)}
$$

which implies

$$
\left|a_{3}\right| \leq \frac{\alpha(\gamma+2)(\gamma+1)^{2}}{(\mu+1)(\mu \gamma-\mu+4 \gamma+2)} .
$$

This completes the proof of theorem.

If we take $\gamma=\mu$, in the Theorem 2.2, we obtain following corollary.

Corollary 2.3. Let $f(z)$ given by (1.1) be in the class $\mathcal{C}_{\Sigma}^{\mu, \mu}(\alpha)(0<\alpha \leq 1)$. Then

$$
\left|a_{2}\right| \leq \alpha \quad \text { and } \quad\left|a_{3}\right| \leq \frac{2 \alpha}{(\gamma+1)^{2}} .
$$




\section{Coefficient estimates for the function class $\mathcal{C}_{\Sigma}^{\mu, \gamma}(\beta)$}

Definition 3.1. A function $f(z)$ given by (1.1) is said to be in the class $\mathcal{C}_{\Sigma}^{\mu, \gamma}(\beta)$ $(0<\mu \leq 1 ; 0<\gamma \leq 1 ; \mu \geq \gamma ; 0 \leq \mu-\gamma<1)$ if the following conditions are satisfied:

$$
f \in \Sigma \quad \text { and } \quad \operatorname{Re}\left\{1+\frac{z(\mathfrak{T} f)^{\prime \prime}(z)}{\mathfrak{T} f^{\prime}(z)}\right\}>\beta \quad(0 \leq \beta<1, z \in \mathbb{U})
$$

and

$$
\operatorname{Re}\left\{1+\frac{w(\mathfrak{T} g)^{\prime \prime}(w)}{\mathfrak{T} g^{\prime}(w)}\right\}>\beta \quad(0 \leq \beta<1, w \in \mathbb{U})
$$

where the function $g$ is inverse of the function $f$ given by (1.2).

For $\gamma=\mu$, the class of $\mathcal{C}_{\Sigma}^{\mu, \gamma}(\beta)$ is reduced to $C_{\Sigma}(\beta)$ of bi-convex of order $\beta$ $(0 \leq \beta<1)$, which is introduced by Brannan and Taha [5], [6].

Theorem 3.2. If $f(z)$ given by (1.1) be in the class $\mathcal{C}_{\Sigma}^{\mu, \gamma}(\beta)$, then

$$
\left|a_{2}\right| \leq \sqrt{\frac{(1-\beta)(\gamma+1)^{2}(\gamma+2)}{(\mu+1)(\mu \gamma-\mu+4 \gamma+2)}}
$$

and

$$
\left|a_{3}\right| \leq \frac{(1-\beta)(\gamma+1)^{2}(\gamma+2)}{(\mu+1)(\mu \gamma-\mu+4 \gamma+2)}
$$

Proof. The inequalities in (3.1) and (3.2) can be written in the following forms :

$$
1+\frac{z(\mathfrak{T} f)^{\prime \prime}(z)}{\mathfrak{T} f^{\prime}(z)}=\beta+(1-\beta) p(z)
$$

and

$$
1+\frac{w(\mathfrak{T} g)^{\prime \prime}(w)}{\mathfrak{T} g^{\prime}(w)}=\beta+(1-\beta) q(w)
$$

where $p(z)$ and $q(w)$ have the forms (2.7) and (2.8), respectively. As in the proof of Theorem 2.2 , by equating coefficients (3.5) and (3.6) yields,

$$
\begin{gathered}
2 \frac{\mu+1}{\gamma+1} a_{2}=(1-\beta) p_{1} \\
\frac{6(\mu+2)(\mu+1)}{(\gamma+2)(\gamma+1)} a_{3}-4\left(\frac{\mu+1}{\gamma+1}\right)^{2} a_{2}^{2}=(1-\beta) p_{2} \\
-2 \frac{\mu+1}{\gamma+1} a_{2}=(1-\beta) q_{1}
\end{gathered}
$$

and

$$
\frac{6(\mu+2)(\mu+1)}{(\gamma+2)(\gamma+1)}\left(2 a_{2}^{2}-a_{3}\right)-4\left(\frac{\mu+1}{\gamma+1}\right)^{2} a_{2}^{2}=(1-\beta) q_{2} .
$$

From (3.7) and (3.9) we get

$$
p_{1}=-q_{1}
$$

and

$$
8\left(\frac{\mu+1}{\gamma+1}\right)^{2} a_{2}^{2}=(1-\beta)^{2}\left(p_{1}^{2}+q_{1}^{2}\right)
$$


Also from (3.8) and (3.10) we obtain

$$
\frac{4(\mu+1)(\mu \gamma-\mu+4 \gamma+2)}{(\gamma+1)^{2}(\gamma+2)} a_{2}^{2}=(1-\beta)\left(p_{2}+q_{2}\right) .
$$

Thus, clearly we have

$$
\left|a_{2}\right|^{2} \leq \frac{(1-\beta)(\gamma+1)^{2}(\gamma+2)}{4(\mu+1)(\mu \gamma-\mu+4 \gamma+2)}\left(\left|p_{2}\right|+\left|q_{2}\right|\right) .
$$

Applying the Carathéodory Lemma for the coefficients $p_{2}$ and $q_{2}$ we find the bound on $\left|a_{2}\right|$ as asserted in (3.3).

In order to find the bound on $\left|a_{3}\right|$, we multiply

$$
\frac{12(\mu+2)(\mu+1)}{(\gamma+2)(\gamma+1)}-\frac{4(\mu+1)^{2}}{(\gamma+1)^{2}} \text { and } \frac{4(\mu+1)^{2}}{(\gamma+1)^{2}}
$$

to the relations (3.8) and (3.10) respectively and on adding them we obtain:

$$
\begin{gathered}
\left\{\frac{24(\mu+2)(\mu+1)^{2}(\mu \gamma-\mu+4 \gamma+2)}{(\gamma+1)^{3}(\gamma+2)^{2}}\right\} a_{3} \\
=(1-\beta)\left\{\frac{4(\mu+1)(2 \mu \gamma+\mu+5 \gamma+4)}{(\gamma+2)(\gamma+1)^{2}} p_{2}+\frac{4(\mu+1)^{2}}{(\gamma+1)^{2}} q_{2}\right\} .
\end{gathered}
$$

Taking the absolute value of (3.15) and applying the Carathéodory Lemma for the coefficients $p_{2}, q_{2}$ we find

$$
\left|a_{3}\right| \leq \frac{(1-\beta)(\gamma+1)^{2}(\gamma+2)}{(\mu+1)(\mu \gamma-\mu+4 \gamma+2)}
$$

which is asserted in (3.4).

If we take $\gamma=\mu$, in the Theorem 3.2, we obtain following corollary.

Corollary 3.3. [5], [6] Let $f(z)$ given by (1.1) belong to $C_{\Sigma}(\beta)(0 \leq \beta<1)$. Then

$$
\left|a_{2}\right| \leq \sqrt{1-\beta} \quad \text { and } \quad\left|a_{3}\right| \leq 1-\beta
$$

\section{References}

[1] Akın, G., Sümer Eker, S., Coefficient estimates for a certain class of analytic and bi-univalent functions defined by fractional derivative, C.R. Math. Acad. Sci. Paris, 352(2014), 1005-1010.

[2] Ali, R.M., Lee, S.K., Ravichandran, V., Supramaniam, S., Coefficient estimates for biunivalent Ma-Minda starlike and convex functions, Appl. Math. Lett., 25(2012), 344-351.

[3] Caglar, M., Deniz, E., Srivastava, H.M., Second Hankel determinant for certain subclasses of bi-univalent functions, Turkish J. Math., 41(2017), 694-706.

[4] Deniz, E., Certain subclasses of bi-univalent functions satisfying subordinate conditions, J. Class. Anal., 2(2013), 49-60.

[5] Brannan, D.A., Taha, T.S., On some classes of bi-univalent functions, Stud. Univ. Babeş-Bolyai Math., 31(1986), no. 2, 70-77. 
[6] Brannan, D.A., Taha, T.S., On some classes of bi-univalent functions, in: S.M. Mazhar, A. Hamoui, N.S. Faour (Eds.), Mathematical Analysis and Its Applications, Kuwait; February 18-21, 1985; in: KFAS Proceedings Series, vol. 3, Pergamon Press, Elsevier Science Limited, Oxford, 1988, pp. 53-60.

[7] Duren, P.L., Univalent Functions, in: Grundlehren der Mathematischen Wissenschaften, Band 259, Springer-Verlag, New York, Berlin, Heidelberg and Tokyo, 1983.

[8] Esa, Z., Kilicman, A., Ibrahim, R.W., Ismail M.R., Husain, S.K.S., Application of Modified Complex Tremblay Operator, AIP Conference Proceedings 1739, 020059 (2016), http://doi.org/10.1063/1.4952539.

[9] Ibrahim, R.W., Jahangiri, J.M., Boundary fractional differential equation in a complex domain, Bound. Value Probl., 2014, Art. ID 66: 1-11.

[10] Kumar, S.S., Kumar V., Ravichandran, V., Estimates for the initial coefficients of biunivalent functions, Tamsui Oxf. J. Inf. Math. Sci., 29(2013), 487-504.

[11] Lewin, M., On a coefficient problem for bi-univalent functions, Proc. Amer. Math. Soc., 18(1967), 63-68.

[12] Magesh, N., Yamini, J., Coefficient bounds for a certain subclass of bi-univalent functions, Internat. Math. Forum, 27(2013), 1337-1344.

[13] Owa, S., On the distortion theorems I, Kyungpook Math. J., 18(1978), 53-59.

[14] Owa, S., Srivastava, H.M., Univalent and starlike generalized hypergeometric functions, Canad. J. Math., 39(1987), 1057-1077.

[15] Pommerenke, Ch., Univalent Functions Göttingen, Vandenhoeck Ruprecht, 1975.

[16] Rudin, W., Real and Complex Analysis, McGraw-Hill Education, 3rd edition, 1986.

[17] Srivastava, H.M., Some inequalities and other results associated with certain subclasses of univalent and bi-univalent analytic functions, in: Nonlinear Analysis: Stability; Approximation; and Inequalities (Panos M. Pardalos,Pando G. Georgiev and Hari M. Srivastava, Eds.), Springer Series on Optimization and Its Applications, Vol. 68, Springer-Verlag, Berlin, Heidelberg and New York, 2012, 607-630.

[18] Srivastava, H.M., Bansal, D., Coefficient estimates for a subclass of analytic and biunivalent functions, J. Egyptian Math. Soc., 23(2015), 242-246.

[19] Srivastava, H.M., Mishra, A.K., Gochhayat, P., Certain subclasses of analytic and biunivalent functions, Appl. Math. Lett., 23(2010), 1188-1192.

[20] Srivastava, H.M., Owa, S., Some characterization and distortion theorems involving fractional calculus, linear operators and certain subclasses of analytic functions, Nagoya Math. J., 106(1987), 1-28.

[21] Srivastava, H.M., Owa, S., Univalent Functions, Fractional Calculus, and Their Applications, Halsted Press, Ellis Horwood Limited, Chichester and John Wiley and Sons, New York, Chichester, Brisbane and Toronto, 1989.

[22] Srivastava, H.M., Sümer Eker, S., Ali, R.M., Coefficient bounds for a certain class of analytic and bi-univalent functions, Filomat, 29(2015), 1839-1845.

[23] Sümer Eker, S., Coefficient bounds for subclasses of m-fold symmetric bi-univalent functions, Turkish J. Math., 40(2016), 641-646.

[24] Sümer Eker, S., Coefficient estimates for new subclasses of m-fold symmetric bi-univalent functions, Theory Appl. Math. Comput. Sci., 6(2)(2016), 103-109.

[25] Taha, T.S., Topics in Univalent Function Theory, Ph. D. Thesis, University of London, 1981. 
[26] Tremblay, R. , Une Contribution 'a la Théorie de la Dérivée Fractionnaire, Ph. D. Thesis, Laval University, Québec, 1974.

Sevtap Sümer Eker

Dicle University, Faculty of Science

Department of Mathematics

21280 Diyarbakır, Turkey

e-mail: sevtaps@dicle.edu.tr

Bilal Şeker

Dicle University, Faculty of Science

Department of Mathematics

21280 Diyarbakır, Turkey

e-mail: bilalseker1980@gmail.com 